\title{
Executive dysfunction in delayed radiation-induced leukoencephalopathy
}

\author{
Alexandre Henri-Bhargava MDCM, Morris Freedman MD
}

$\mathrm{W}$ e admitted a 52-year-old woman to hospital for evaluation of a four-year history of progressive cognitive impairment, irritable affect, gait impairment and incontinence. Thirty years earlier, she had undergone surgery and whole-brain radiotherapy (50 Gy in 25 fractions) for a right frontal oligodendroglioma. Her neurologic examination showed paratonia, primitive reflexes, hyperreflexia and difficulty initiating gait, despite preserved leg strength. Cognitive testing demonstrated impaired memory, naming and comprehension, as well as severe executive dysfunction as illustrated by impaired clock drawing ${ }^{1}$ (Figure 1). Magnetic resonance imaging of the brain showed postoperative changes and marked, abnormal, $T_{2}$ signal hyperintensity involving white matter of frontal and parietal lobes bilaterally (Appendix 1, available at www.cmaj.ca/lookup/suppl/doi: 10.1503/cmaj.101879/-/DC1).

Based on the clinical and radiologic findings, we diagnosed delayed radiation-induced leukoencephalopathy. This disease may occur months to several years following therapeutic cranial irradiation, and incidence estimates vary widely from $2 \%$ to $50 \% .^{2,3}$ Symptoms include progressive mental slowing and cognitive decline leading to frontal-subcortical dementia. It may be accompanied by gait difficulties and urinary incontinence. Magnetic resonance imaging will initially show progressive subcortical atrophy, followed by periventricular white matter changes and finally confluent white matter abnormalities, as in our patient. The degree of abnormality on imaging correlates with $\operatorname{cog}$ nitive deficits. ${ }^{3}$

The risk of radiation-induced leukoencephalopathy appears to be related to the total dose of radiation ( $>45-50 \mathrm{~Gy}$ ), the concomitant use of chemotherapy, age at the time of radiotherapy and coexisting white matter disease such as mul-

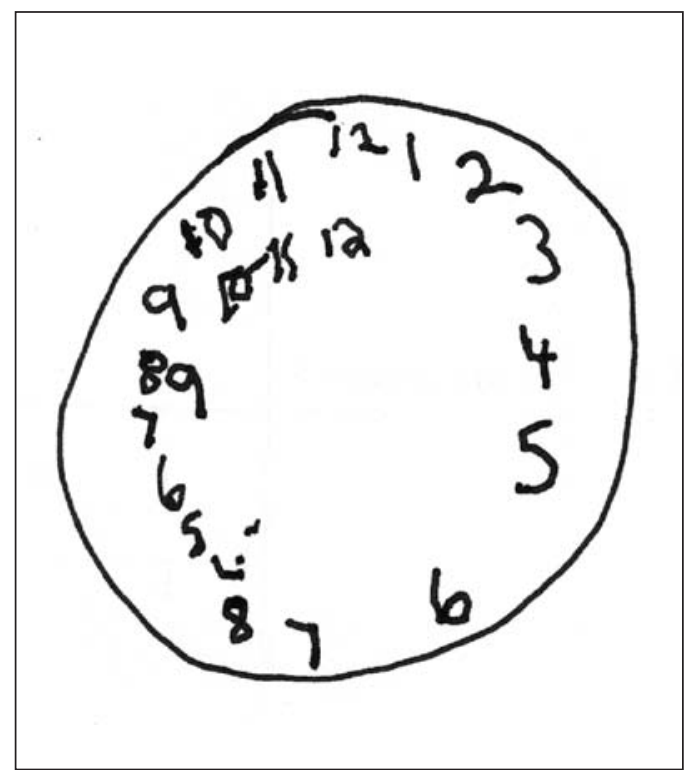

Figure 1: Clock drawn by a 52-year-old woman with delayed radiation-induced leukoencephalopathy. Performance was suggestive of executive system dysfunction. Instructions were to set the clock at "ten past eleven." The patient performed very slowly and showed poor planning in number placement. She lost track after writing " 8 " (lower left), resumed at "4" and completed numbers to "12." She wrote "10-11" after being cued, and then added an extra "9" and "12."

tiple sclerosis or leukoaraiosis. ${ }^{2}$ Treatment is supportive, because no specific therapies are known to reverse the usually progressive course of this illness.

\section{References}

1. Freedman M, Leach L, Kaplan E, et al. Clock drawing: a neuropsychological approach. New York (NY): Oxford University Press; 1994

2. Taphoorn MJ, Bromberg JE. Neurological effects of therapeutic irradiation. Contin Lifelong Learn Neurol 2005;11:93-115.

3. Johannesen TB, Lien HH, Hole KH, et al. Radiological and clinical assessment of long-term brain tumour survivors after radiotherapy. Radiother Oncol 2003;69:169-76.
Competing interests: None declared by Alexandre Henri-Bhargava. Morris Freedman is listed on a provisional patent related to diagnosis of Alzheimer disease versus

frontotemporal dementia. $\mathrm{He}$ has received royalties for a book on clock drawing for cognitive assessment. ${ }^{1}$

This article has been peer reviewed.

Affiliations: From the Department of Medicine, Division of Neurology at Baycrest (Henri-Bhargava, Freedman), Mount Sinai Hospital (Freedman) and University of Toronto (Henri-Bhargava, Freedman); and Rotman Research Institute, Baycrest (HenriBhargava, Freedman) Toronto, Ont.

Acknowledgements: Alexandre Henri-Bhargava has been funded in part by a fellowship from Eli Lilly Canada. Morris Freedman is supported in part by the Saul A. Silverman Family Foundation as part of the

Canada International

Scientific Exchange

Program.

Correspondence to: Dr. Alexandre HenriBhargava, ahenribhargava @baycrest.org

CMAJ 2011. DOI:10.1503 /cmaj.101879 